\title{
FLORÍSTICA, DIVERSIDADE E DISTRIBUIÇÃO ESPACIAL DAS ESPÉCIES ÁRBOREAS DE UM TRECHO DE FLORESTA ESTACIONAL SEMIDECIDUAL DA SERRA DA CONCÓRDIA, RJ
}

\author{
Welington Kiffer de Freitas ${ }^{1}$, Luís Mauro Sampaio Magalhães ${ }^{2}$ \\ ${ }^{1}$ Eng. Florestal, Dr., Depto. de Eng. de Produção, UFF, Volta Redonda, RJ, Brasil - wkfreitas@gmail.com; \\ ${ }^{2}$ Eng. Florestal, Dr., Depto. de Ciências Ambientais, Instituto de Florestas, UFRRJ, Seropédica, RJ, Brasil - 1.mauro@terra.com.br \\ Recebido para publicação: 28/01/2013 - Aceito para publicação: 08/12/2013
}

\begin{abstract}
Resumo
O estudo foi realizado na borda de um fragmento de Floresta Estacional Semidecidual na Serra da Concórdia, Vale do Paraíba do Sul (RJ). Foram instaladas amostras em dois sítios, cada uma com 10 subparcelas de $100 \mathrm{~m}^{2}$, totalizando 0,2 ha. Foi coletado o material botânico das árvores com Diâmetro à Altura do Peito (DAP) maior ou igual a $5,0 \mathrm{~cm}$. Foram amostrados 247 indivíduos, pertencentes a 43 espécies e 23 famílias. A síndrome de dispersão zoocórica, com $51,8 \%$ dos indivíduos e $48,8 \%$ das espécies e o grupo ecológico das pioneiras, com $33,3 \%$ dos indivíduos e $29,7 \%$ das espécies, foram os que apresentaram maior ocorrência. Foram analisados os índices de diversidade de Shannon (H') e de Pielou (J), além do padrão de distribuição das espécies, através do índice de Morisita $\left(\mathrm{I}_{\mathrm{d}}\right)$. Os valores para os dois primeiros índices foram, respectivamente, 3,15 e 0,84 . O padrão de distribuição espacial das espécies revelou que $34,1 \%$ das espécies apresentaram padrão de dispersão agregado, 29,5\% uniforme e 6,8 \% aleatório. A Serra da Concórdia é considerada um importante remanescente da Mata Atlântica do médio Paraíba do Sul, o que reforça a adoção de medidas de conservação desse grande repositório da biodiversidade regional.

Palavras-chave: Ecologia florestal; floresta de encosta; índice de Morisita; Mata Atlântica.
\end{abstract}

\begin{abstract}
Floristic, diversity and spatial distributions of tree species in a Semideciduous Forest site in Sierra of Concórdia, RJ, Brazil. This research was conducted at the edge of a fragment of semideciduous forest in Serra da Concórdia, Valley of Paraíba do Sul ( RJ ). Samplings were installed in two sites, with 10 parcels of $100 \mathrm{~m}^{2}$ each, totaling 0.2 ha. The botanical material was collected from trees with diameter at breast height $(\mathrm{DBH})$ greater than or equal to $5.0 \mathrm{~cm}$. The results revealed 247 trees belonging to 43 species and 23 families. The number of trees and of species were higher for dispersion syndrome of zoochory ( $51.8 \%$ of trees and $48.8 \%$ of the species) and for ecological group of pioneers $(33.3 \%$ of subjects and $29.7 \%$ of the species). The diversity index of Shannon $\left(\mathrm{H}^{\prime}\right)$, of Pielou $(\mathrm{J})$, and the distribution pattern of the species by the Morisita index (Id) were analyzed. The values for the first two indexes were respectively 3.15 and 0.84 . The spatial distribution of the species revealed that 34.1 $\%$ had aggregated dispersion pattern, $29.5 \%$ uniform distribution, and $6.8 \%$ presented random distribution. The forest of the Serra da Concórdia is considered an important remnant of the Atlantic Forest in the middle Paraíba do Sul, which reinforces the adoption of conservation measures for that great repository of regional biodiversity.
\end{abstract}

Keywords: Forest ecology; slope forest; Morisita index; Atlantic Forest.

\section{INTRODUÇÃO}

O bioma da Floresta Atlântica é considerado um centro de diversidade e de endemismo para várias espécies de plantas vasculares (PEIXOTO; GENTRY, 1990) e sua conservação depende fundamentalmente da proteção das diferentes fisionomias vegetais que o constituem. Neste sentido, o levantamento da composição florística tem se mostrado uma ferramenta útil, pois permite conhecer o estado de degradação ou conservação de áreas deste bioma, incluindo os estudos das espécies regionais 
mais importantes e do papel exercido por cada uma dentro da comunidade (GROMBONE et al., 1990; BÓREM; OLIVEIRA FILHO, 2002; GUEDES-BRUNI, 1998).

Recentemente, alguns trabalhos têm trazido contribuições a respeito destes ecossistemas, como nos resultados sobre remanescentes de floresta sobre tabuleiro terciário, Município de São Francisco do Itabapoana (SILVA; NASCIMENTO, 2001); em trecho florestal da bacia hidrográfica de São Domingos, São José de Ubá (DAN et al., 2010); na planície aluvionar nas margens do rio Guandu, Seropédica (RODRIGUES; MAGALHÃES, 2011); em trecho de Floresta Ombrófila Densa Submontana, Nova Iguaçu (PINTO SOBRINHO et al., 2010); e em trechos de Florestas Estacionais Semideciduais na região do vale do rio Paraíba do Sul (SPOLIDORO, 2001; SOUZA et al. 2007; FERNANDES et al., 2012). Os estudos florísticos, além de gerar informações sobre classificação e distribuição taxonômica no nível de família, gênero e espécie de uma comunidade vegetal, também servem de subsídio pra investigações sobre atributos ecológicos das espécies, como formações de grupos ecológicos e síndromes de dispersão, além de permitirem estudos importantes para o manejo, como o de padrão de distribuição das espécies (FREITAS; MAGALHÃES, 2012).

O padrão de distribuição espacial das espécies arbóreas é uma questão chave para estudos em ecologia florestal, sendo vital para entender como determinada espécie utiliza os recursos disponíveis e, ainda, porque é atribuído o sucesso do estabelecimento e da reprodução de determinadas plantas (SILVA et al., 2009). Conforme Crawley (1997), a distribuição espacial de indivíduos maduros reflete o padrão espacial de recrutamento e a influência de fatores de mortalidade, tornando-se uma ferramenta útil para subsidiar a estratégia de manejo e/ou conservação de uma população ou de uma comunidade florestal. Hutchinson (1953) foi um dos primeiros ecólogos a reconhecer fatores gerais que podem afetar a distribuição de uma população incluindo: (1) fatores vetoriais provenientes do ambiente exterior (e.g., vento, corrente de água); (2) fatores reprodutivos; (3) fatores devido a interações intraespecíficas (ex. competição); e (4) fatores aleatórios resultantes de variação ao acaso dos fatores considerados. Os efeitos desses movimentos podem determinar diferentes padrões de distribuição, sendo eles: agregado, uniforme ou aleatório (TOWNSEND et al. 2010).

Nesse contexto, o objetivo geral deste trabalho foi o de avaliar a composição florística e o estágio sucessional de um trecho de Floresta Estacional Semidecidual de encosta na Serra da Concórdia, na região do vale do Paraíba do Sul, RJ, utilizando-se o índice de diversidade de Shannon (H'), a uniformidade das espécies através do índice de Pielou (J') e o padrão de distribuição espacial das espécies pelo índice de Morisita $\left(\mathrm{I}_{\mathrm{d}}\right)$. Com a avaliação dos diferentes tipos de distribuição das espécies, se espera avançar no conhecimento necessário para o manejo da floresta examinada, contribuindo para a sua conservação e para a manutenção dos benefícios desta cobertura, como a proteção à fauna local, ao solo e aos recursos hídricos.

\section{MATERIAL E MÉTODOS}

O trabalho foi realizado no Santuário da Vida Silvestre da Serra da Concórdia, divisa dos municípios de Barra do Piraí e Valença, RJ, setor médio da bacia do rio Paraíba do Sul, situado nas coordenadas $22^{\circ} 22^{\prime} 20^{\prime \prime} \mathrm{S}$ e $43^{\circ} 47^{\prime} 23^{\prime \prime} \mathrm{O}$, a uma altitude média de $650 \mathrm{~m}$, com uma área de cobertura florestal de aproximadamente 220 ha (CALDAS, 2006).

Pertence à região fitoecológica denominada Floresta Estacional Semidecidual Montana, devido à ocorrência de déficit hídrico durante a estação seca e à consequente adaptação fisiológica dos elementos arbóreos dominantes, que no conjunto florestal apresentam queda foliar entre 20\% a 50\% (INSTITUTO BRASILEIRO DE GEOGRAFIA E ESTATÍSTICA (IBGE), 1992).

O clima da região corresponde ao tipo Cwa, conforme a classificação de Köppen (1948), mesotérmico úmido, com média do mês mais frio inferior a $18^{\circ} \mathrm{C}$ e mês mais quente com temperatura superior a $22^{\circ} \mathrm{C}$, com estação seca no inverno. A pluviosidade média anual é de $1.212 \mathrm{~mm}$ (CALDAS, 2006).

Foram instaladas parcelas de $20 \mathrm{~m}$ x $50 \mathrm{~m}$, em dois sítios de uma mesma topossequência, sendo cada uma, dividida em 10 subparcelas, com $10 \mathrm{~m}$ x $10 \mathrm{~m}$, totalizando 0,2 ha. Nas 20 subparcelas foram registradas todas as espécies arbóreas vivas, com Circunferência à Altura do Peito (CAP) igual ou maior a $15,71 \mathrm{~cm}$ (ou DAP igual ou maior a $5 \mathrm{~cm}$ ). De cada indivíduo foi medido o CAP e a altura total (com auxílio da vara do podão) e coletado material botânico. 
Todo material coletado foi submetido à secagem em estufa, para posterior identificação através de caracteres morfológicos, comparando com exsicatas, consulta bibliográfica e auxílio de especialistas. O material fértil coletado encontra-se depositado no herbário da UFRRJ (RBR), com números de registro de 29.826 a 29.849. O sistema de classificação adotado foi o do Angiosperm Phylogeny Group - APG III (APG III, 2009). A família Fabaceae foi subdividida em três subfamílias: Caesalpinioideae, Papilionideae e Mimosoideae (POLHILL et al. 1981). A validação dos nomes das espécies e a exclusão das sinonímias botânicas foram obtidas através de consulta aos sites do Missouri Botanical Garden (MOBOT, 2012) e do International Plant Names Index (IPNI, 2012).

Nesse estudo foram ainda aplicados os seguintes índices ecológicos (FREITAS; MAGALHÃES, 2012; POOLE, 1974): Índice de diversidade de Shannon (H'), Índice de Pielou (J) e Índice de Morisita. A significância do Índice de Dispersão de Morisita $\left(\mathrm{I}_{\mathrm{d}} \neq 1\right)$ foi testada através do teste $\mathrm{F}$ para significância do $\mathrm{I}_{\mathrm{d}}(\mathrm{gl}=\mathrm{n}-1 ; \mathrm{p}<0,05)($ POOLE, 1974).

\section{RESULTADOS E DISCUSSÃO}

Esse estudo registrou a presença de 247 indivíduos pertencentes a 43 espécies, 39 gêneros e 23 famílias botânicas (Tabela 1). Do total de espécies, seis foram identificadas no nível de gênero.

Tabela 1. Lista das espécies arbóreas amostradas em um fragmento florestal da Serra da Concórdia, municípios de Valença e Barra do Piraí, RJ, em ordem alfabética, por família.

Table 1. List of tree species sampled in a forest fragment of the Serra da Concórdia (Valença and Barra do Piraí, RJ, Brazil), in alphabetical order by family.

\begin{tabular}{|c|c|c|}
\hline Família & Espécies & $\begin{array}{l}\text { Número de } \\
\text { indivíduos }\end{array}$ \\
\hline \multirow[t]{3}{*}{ ANNONACEAE } & Rollinia sericeae (R.E. Fries) R. E. Fries & 7 \\
\hline & Rollinia sp & 2 \\
\hline & Tabernamontana laeta Mart & 1 \\
\hline APOCYNACEAE & Rauvolfia sp & 6 \\
\hline \multirow[t]{2}{*}{ ASTERACEAE } & Vernonia discolor (Spreng.) Less. & 2 \\
\hline & Vernonia puberula Less. & 1 \\
\hline \multirow[t]{2}{*}{ BIGNONIACEAE } & Handroanthus albus (Cham.) Mattos & 1 \\
\hline & Sparattosperma leucanthum (Vell.) K. Schum. & 2 \\
\hline CELASTRACEAE & Maytenus brasiliensis Mart. & 1 \\
\hline CUNONIACEAE & Lamanonia ternata Vell. & 1 \\
\hline EUPHORBIACEAE & Croton floribundus Spreng. & 6 \\
\hline \multirow[t]{6}{*}{ FABACEAE - MIMIMOSOIDEAE } & Acacia poliphylla DC. & 1 \\
\hline & Anadenanthera colubrina (Vell) Brenan. & 4 \\
\hline & Inga sp & 1 \\
\hline & Mimosa artemisiana Heringer et Paula & 17 \\
\hline & Piptadenia gonoacantha (Mart.) Macbr. & 41 \\
\hline & Piptadenia paniculata Mart. & 4 \\
\hline \multirow[t]{2}{*}{ FABACEAE - PAPILIONIDEAE } & Dalbergia $\mathrm{sp}$ & 1 \\
\hline & Machaerium hirtum (Vell.) Stellfeld & 16 \\
\hline \multirow[t]{3}{*}{ LAURACEAE } & Endlicheria paniculata (Spreng) Macbr. & 1 \\
\hline & Nectandra membranacea Sw. & 4 \\
\hline & Ocotea schwackeana Mez & 3 \\
\hline LECYTHIDACEAE & Cariniana estrellensis (Raddi) Kuntze. & 2 \\
\hline \multirow[t]{2}{*}{ MALVACEAE } & Ceiba speciosa St. Hil. & 2 \\
\hline & Luehea grandiflora Mart et Zucc. & 3 \\
\hline MELASTOMATACEAE & Tibouchina granulosa Cogn. & 1 \\
\hline \multirow[t]{2}{*}{ MELIACEAE } & Cabralea canjerana (Vell.) Mart. & 5 \\
\hline & Guarea kunthiana A. Juss & 7 \\
\hline MORACEAE & Ficus insipida Wild. & 1 \\
\hline MYRTACEAE & Campomanesia sp & 4 \\
\hline
\end{tabular}




\begin{tabular}{llc}
\hline \multirow{2}{*}{ NYCTAGINACEAE } & Myrcia rostrata DC. & 2 \\
& Guapira opposita (Vell.) Reitz. & 16 \\
PROTEACEAE & Pisonia cf. glabra Ficher & 2 \\
RUBIACEAE & Roupala sp & 1 \\
SALICACEAE & Psychotria caudata M. Gomes & 14 \\
& Casearia sylvestris Sw. & 14 \\
SAPINDACEAE & Xylosma prockia (Turcz.) Turcz. & 1 \\
& Allophyllus aff. edullis (A. St. Hil) Radlk. & 21 \\
SOLANACEAE & Cupania oblongifolia Cambess. & 3 \\
& Aureliana fasciculata (Vell.) Sendtn. & 2 \\
URTICACEAE & Solanum argentum Dun. & 4 \\
\hline Total & Solanum granulo-leprosum Dun. & 2 \\
\hline
\end{tabular}

Embora a suficiência amostral seja um conceito muito utilizado em fitossociologia, não existe nenhum método inteiramente satisfatório para determiná-lo (DIETVORST et al, 1982). O método mais comum é o uso de curvas espécie-área (CAIN, 1938), mesmo considerando o problema de que as curvas raramente atingem o nível de saturação, e a determinação da suficiência do tamanhı da amostra é geralmente baseada em análises subjetivas (DIETVORST et al., 1982).

A suficiência amostral foi testada pela curva espécie-área (Figura 1). O modelo matemático que melhor explicou os dados foi o polinomial de terceira ordem, através da equação $\mathrm{y}=0,0036 \mathrm{x}^{3}-0,1901 \mathrm{x}^{2}$ $+4,3717 \mathrm{x}+5,2062$, com alta significância no parâmetro do ajuste da curva: $\mathrm{R}^{2}>0,96$. Scudeller et al. (2001) afirmam que ecossistemas florestais tropicais possuem muitas espécies com baixa densidade e poucas espécies em maior densidade, sendo esse um dos fatores que permitem a coexistência de tantas espécies em um mesmo local. Por esse motivo, Lamprecht (1990) salienta que nas florestas tropicais a curva estaria adequada, quando um aumento de $10 \%$ na área amostrada, correspondesse a um aumento de $10 \%$ no número de espécies. Neste caso, nas duas últimas parcelas ( $10 \%$ da área amostrada) foi observado o ingresso de apenas uma espécie nova (2,27\%). Todavia, para a estabilização efetiva da curva seria necessária a instalação de mais unidades amostrais.

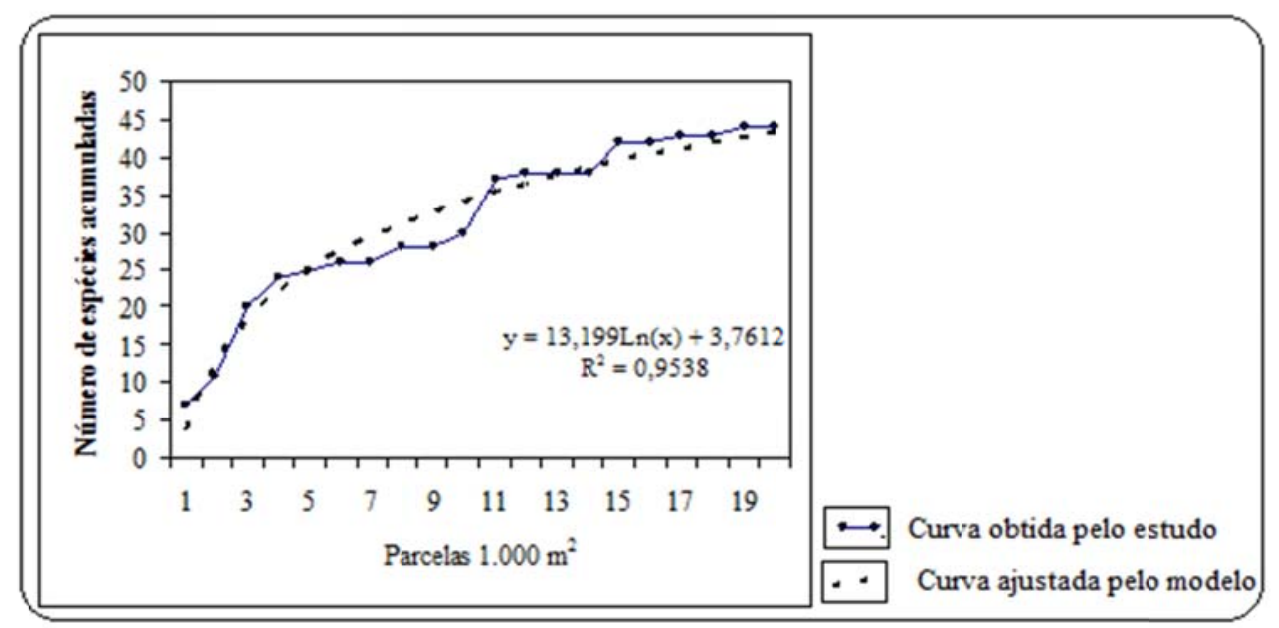

Figura 1. Curva cumulativa de espécies com DAP $>5,0 \mathrm{~cm}$, versus área amostrada no trecho de Floresta Estacional Semidecidual localizado na Serra da Concórdia, municípios de Valença e Barra do Piraí, RJ.

Figure 1. Cumulative curve of species with $\mathrm{DBH}>5,0 \mathrm{~cm}$, versus the sampled area on two sites of semideciduous forest located in Serra da Concórdia (Valença and Barra do Piraí, RJ, Brazil). 
Outros estudos realizados em Florestas Estacionais Semideciduais, utilizando método de parcelas e critério de inclusão de DAP $\geq 5 \mathrm{~cm}$, realizados por Durigan et al. (2000) em Gália (SP) e por Fonseca e Rodrigues (2000) em Botucatu (SP), encontraram resultados pouco superiores para os parâmetros apresentados: 62 espécies e 28 famílias e 61 espécies e 31 famílias, respectivamente. Todavia, em termos de densidade, o presente estudo registrou um total de 1.235 indivíduos . ha ${ }^{-1}$, valor superior ao encontrado por Durigan et al. (2000) (1.080 ind . ha ${ }^{-1}$ ) e por Fonseca e Rodrigues (2000) (1.104 ind . ha ${ }^{-1}$ ).

No Triangulo Mineiro, sudeste do Brasil, Lopes et al. (2012) examinaram os padrões florísticos de dez sítios de Florestas semi-deciduais, considerando o critério de inclusão de DAP $\geq 4,8 \mathrm{~cm}$, encontrando 242 espécies arbóreas, 163 gêneros e 58 famílias. Fabaceae (38 espécies) e Myrtaceae (20 espécies) foram as famílias com maior número de espécies. Os autores revelaram que as similaridades entre os fragmentos estudados estavam ligadas principalmente ao estágio sucessional da comunidade.

Araújo et al. (1997) ao analisarem um fragmento florestal semidecidual no Triangulo Mineiro, na região de Araguari, encontraram 1.827 indivíduos de 113 espécies em 46 famílias, ou uma densidade 1.552 ind.ha ${ }^{-1}$, considerando todas as árvores com DAP $\geq 10 \mathrm{~cm}$, encontradas em 60 parcelas distribuídas em um fragmento com cerca de 11 ha e integralizando uma amostragem de 1,2 ha. Da mesma forma, Rodrigues et al. (2003), no sul de Minas Gerais (Luminárias), registraram 2.343 indivíduos, pertencentes a 159 espécies e 50 famílias, em uma área amostral de 1,0 ha. Souza et al.(2007), analisando um trecho de Floresta Semidecidual, também localizado no médio vale do rio Paraíba do Sul, na Área de Relevante Interesse Ecológico (ARIE) da Cicuta, Volta Redonda (RJ), com amostragem de 0,3 ha, porém com critério de inclusão de DAP $\geq 2,5 \mathrm{~cm}$, encontraram valores bem superiores; com 46 famílias, 184 espécies e densidade de 3.227 ind . ha ${ }^{-1}$, incluindo árvores e arbustos.

Outros estudos têm se debruçado sobre florestas estacionais semideciduais, com indicações importantes para a compreensão da sua distribuição. Prado e Gibbs (1993) relatam a ocorrência de uma estreita ligação entre a vegetação da Caatinga e do Chaco argentino por meio de espécies que atravessam o Brasil, ocorrendo em áreas de matas sazonais da Floresta Attântica (Florestas Estacionais do Brasil Central e da calha do rio Uruguai) e da região do Cerrado. Essa complexa ligação sugere que talvez essa formação vegetal contínua tenha alcançado sua extensão máxima durante um período de clima seco e frio do Pleistoceno, sendo essa teoria de grande valia para a compreensão do padrão de distribuição atual das formações vegetais por meio de ligações florísticas, indicadas pelas espécies de ampla distribuição, e por mudanças climáticas ocorridas no passado.

Oliveira Filho et al. (2006) avaliaram as relações florísticas das florestas sazonalmente secas do leste da América do Sul, com base no padrões de distribuição das espécies. De acordo com esses autores, foi constatado que na área de estudo aproximadamente $22 \%$ dos indivíduos e $25 \%$ das espécies são classificadas como "supertramps", ou seja, espécies que apresentam ampla distribuição em várias fitofisionomias de floresta sob o domínio Atlântico no Brasil.

Além dos resultados já mencionados, as observações da área mostram que a subfamília Fabaceae-mimosoideae apresentou maior número de espécies, com seis espécies, seguidas de Annonacee, Lauraceae e Solanaceae, com três espécies cada; juntas, essas espécies totalizaram mais de $65 \%$ de todas as espécies amostradas.

Com relação ao número de indivíduos por família, quatro famílias totalizam cerca de $60 \%$ de todos os indivíduos amostrados, sendo elas: Fabaceae-mimosoideae (27,3\%), Sapindaceae (10,0\%), Nyctaginaceae (7,2\%), Fabaceae-papilionideae e Urticaceae (6,8\% cada uma). A família Fabaceae também apresentou maior número de indivíduos na ARIE da Cicuta (Volta Redonda, RJ) (SOUZA et al., 2007). Segundo Gentry (1988) e Oliveira-Filho e Fontes (2000), de uma maneira geral, a família Fabaceae é sempre a mais rica em regiões tropicais onde existe uma estação seca bem pronunciada em termos de número de gêneros para os biomas Mata Atlântica, Amazônia e Cerrado. Ecologicamente, o sucesso dessa família tem sido atribuído aos seus métodos de defesa (acúleos, tanino), à eficiência na reprodução, à capacidade de adquirir substâncias essenciais para o crescimento, além do fato que, muitas espécies são capazes de fixar nitrogênio atmosférico através da associação com bactérias do gênero Rhizobium (POLHILL, 1981; MOREIRA, 1994).

Seis espécies somaram mais de $50 \%$ do total de indivíduos. As espécies mais abundantes nesse estudo apresentam comportamento de estágios serais iniciais, típicas colonizadoras e clareiras e das bordas de fragmentos florestais, como as pioneiras (Piptadenia gonoacantha, Cecropia glaziovi, Mimosa artemisiana e Machaerium hirtum) e as secundárias iniciais (Allophyllus aff. Edullis e Guapira opposita). 
Dessas, apenas Piptadenia gonoacantha esteve presente entre as dez espécies mais importantes em trechos de Florestas Semideciduais antropizadas da região do vale do Paraíba (RJ) (SPOLIDORO, 2001; FERNANDES et al., 2012).

Com respeito ao Índice de diversidade de Shannon (H'), foi encontrada uma diversidade relativamente elevada com $\mathrm{H}^{\prime}$ de 3,15 nats.ind $^{-1}$, considerando que o stand examinado sofre efeito de borda. A fragmentação florestal pode causar mudanças sobre a hidrologia regional, a biodiversidade e aumentar o risco de incêndios e favorecer a mortalidade das árvores em uma faixa de até 100 metros da borda dos fragmentos, aumentando os efeitos de borda (FERREIRA ; LAURENCE, 1997). Conforme Zaú (1998), nas bordas é bastante frequente a presença de espécies com características pioneiras e típicas de clareiras (heliófilas), com muitos indivíduos de poucas espécies, características de estado sucessional inicial.

Spolidoro (2001), ao estudar um trecho de Floresta Estacional, submetida a três épocas de pousio (80, 60 e 120 anos), encontrou diversidade superior à desse estudo apenas na última parcela, que teve regeneração natural a partir de área de cultivo de café, sendo esse trecho favorecido, principalmente, pela dificuldade de acesso e tempo de permanência. Ivanauskas et al (1999), encontraram H' superior ao desse estudo, também associado ao longo tempo de pousio. Por outro lado, Fernandes et al (2012) e Durigan et al (2000) observaram menor diversidade, podendo esse fato estar associado ao efeito de borda, tornando o ambiente mais favorável à ocupação de grupo de espécies mais tolerantes, principalmente, à insolação, ao déficit hídrico e à competição. Estudos com áreas amostrais de 0,95 a 1,0 hectare revelaram H' variando de 3,33 a 4,01 nats . ind ${ }^{-1}$, reforçando a importância do efeito de borda sobre a diversidade do estrato arbóreo. Conforme Rolim e Nascimento (1997) além da intensidade amostral, o conhecimento do nível de exclusão de Diâmetro à Altura do Peito (DAP) e o rigor utilizado para a identificação das espécies são fundamentais para a interpretação do índice de Shannon.

A determinação de qual seria a diversidade máxima da comunidade florestal foi calculada através do índice de equitabilidade de Pielou ( $\mathrm{J}$ '), resultando em 0,84 . Na grande maioria dos estudos citados, como Spolidoro (2001) e Ivanauskas et al (1999), a uniformidade das espécies, demonstrada através do índice de Pielou, foi superior 0,78. Este é um índice de uniformidade, e seu resultado é fortemente influenciado pelo número de espécies. Em um contexto regional, o índice J' indica uma concentração relativamente alta de abundâncias em um pequeno número de espécies, as quais dominam a comunidade arbórea.

Através do índice de agregação de espécies de Morisita (Tabela 2), foi possível visualizar a distribuição espacial das espécies dentro da comunidade, o que pode configurar um ponto essencial para a aplicação teórica e como subsídio para o manejo (LEGENDRE; FORTIN, 1989). Nesse estudo 34,1\% das espécies apresentaram padrão de dispersão agregado, 29,5\% uniforme e 6,8\% aleatório.

Nessa comunidade foi registrado um pequeno percentual de espécie com distribuição aleatória, pouco acima de 5\%. Conforme Silva et al. (2009), o padrão aleatório infere que a posição de cada indivíduo é independente a de todos os outros de sua espécie, de tal forma que qualquer um possui a mesma chance de ocorrer em qualquer ponto da área selecionada como é o caso de Piptadenia gonoacantha Allophylus aff. edulis e Machaerium hirtum. Esse padrão reflete uma homogeneidade ambiental, com padrões comportamentais não seletivos, sugerindo interações neutras entre os indivíduos e o ambiente local (CAIN et al., 2011). De acordo com Oliveira e Amaral (2005) a distribuição aleatória é rara em populações vegetais.

O padrão uniforme foi observado em cerca de 30\% das espécies presentes no trecho florestal amostrado. Porém, esse estudo registrou a presença de Croton floribundus (6 ind.), Nectandra membranaceae (4 ind.), Piptadenia paniculata (4 ind.) e Solanum argenteum (4 ind.) (Tabela 2) que podem estar utilizando de fatores controladores da densidade (por exemplo, alelopatia) ou fatores ambientais (características edáficas, posição de vertente e outros) para se estabelecer de forma uniforme (SILVA et al., 2009). Townsend et al. (2010) salientam que algumas espécies mostram-se distribuídas aleatoriamente em ambientes onde recursos se encontram limitados devido à saturação de sítios, promovendo assim, uma severa competição.

$\mathrm{O}$ padrão de distribuição agregado predomina em florestas tropicais, sugerindo uma força de atração, onde a chance de ocorrência de um indivíduo é aumentada pela presença de outros do mesmo taxa ou a pela disponibilidade de um recurso comum (SILVA et al., 2008; CAIN et al., 2011). Nesse estudo, aproximadamente $35 \%$ das espécies apresentaram padrão agregado, dentre elas: Cecropia glaziovi 
(17 indivíduos), Guapira opposita (16 ind.), Psychotria caudata (15 ind.) e Casearia sylvestris (14 ind.) (Tabela 2). De acordo com Townsend et al. (2010) a explicação evolutiva mais simples para a distribuição agregada é que os organismos se agregam, quando e onde encontram condições favoráveis à reprodução e a sua sobrevivência.

Cabe ressaltar que o padrão espacial das espécies pode ocorrer em diferentes escalas simultaneamente (CAIN et al., 2011). Hay et al. (2000) consideraram três escalas principais: macro (biogeográfica), meso (comunidades) e micro (indivíduos dentro de uma comunidade). Nesse estudo foi considerada a micro escala, restringindo-se a compreensão da borda do fragmento florestal.

Tabela 2. Relação das espécies amostradas na Floresta Estacional Semidecidual da Serra da Concórdia, municípios de Valença e Barra do Piraí, RJ, Brasil, com os valores para o índice de Morisita $\left(I_{d}\right)$, "F" calculado e os respectivos padrões de dispersão.

Table 2. List of species sampled in the Seasonal Semideciduous Forest of the Serra da Concórdia (Valença and Barra do Piraí, RJ, Brazil), with values for the index Morisita $\left(\mathrm{I}_{\mathrm{d}}\right)$, calculated value of "F" and respective dispersal patterns.

\begin{tabular}{|c|c|c|c|}
\hline \multirow{2}{*}{ Espécies } & \multicolumn{3}{|c|}{ Padrão de distribuição espacial de Morisita } \\
\hline & Id & F calculado & Classificação \\
\hline Cecropia glaziovi & 17,193 & 16,341 & Agregado* \\
\hline Mimosa artemisiana & 6,176 & 5,359 & Agregado* \\
\hline Guapira opposita & 8,000 & 6,526 & Agregado* \\
\hline Psychotria caudata & 6,857 & 5,316 & Agregado* \\
\hline Casearia sylvestris & 4,615 & 3,474 & Agregado* \\
\hline Guarea kunthiana & 11,490 & 4,293 & Agregado* \\
\hline Rollinia sericea & 5,714 & 2,489 & Agregado* \\
\hline Rauvolfia sp & 4,000 & 1,789 & Agregado* \\
\hline Anadenanthera colubrina & 6,000 & 2,053 & Agregado* \\
\hline Cabralea canjerana & 6,000 & 2,053 & Agregado* \\
\hline Campomanesia sp & 12,000 & 3,313 & Agregado* \\
\hline Luehea grandiflora & 20,000 & 3,000 & Agregado* \\
\hline Ocotea shwackeana & 20,000 & 3,000 & Agregado* \\
\hline Dalbergia sp & 60,000 & 4,105 & Agregado* \\
\hline Piptadenia gonoacantha & 4,599 & 8,766 & Aleatória $^{\text {ns }}$ \\
\hline Allophylus aff. edulis & 6,403 & 7,256 & Aleatória $^{\text {ns }}$ \\
\hline Machaerium hirtum & 13,143 & 13,782 & Aleatória $^{\text {ns }}$ \\
\hline Croton floribundus & 0,000 & $*$ & Uniforme \\
\hline Nectandra membranaceae & 0,000 & $*$ & Uniforme \\
\hline Piptadenia paniculata & 0,000 & $*$ & Uniforme \\
\hline Solanum argenteum & 0,000 & * & Uniforme \\
\hline Cupania oblongifolia & 0,000 & $*$ & Uniforme \\
\hline Cariniana estrellensis & 0,000 & $*$ & Uniforme \\
\hline Ceiba speciosa & 0,000 & $*$ & Uniforme \\
\hline Myrcia rostrata & 0,000 & $*$ & Uniforme \\
\hline Pisonia cf. glabra & 0,000 & $*$ & Uniforme \\
\hline Rollinia $\mathrm{sp}$ & 0,000 & $*$ & Uniforme \\
\hline Solanum granulo-leprosum & 0,000 & $*$ & Uniforme \\
\hline Sparattosperma leucanthum & 0,000 & * & Uniforme \\
\hline Vernonia discolor & 0,000 & $*$ & Uniforme \\
\hline
\end{tabular}

Os resultados obtidos podem auxiliar as medidas de manejo, adotando a microescala, como, por exemplo, a escolha de medidas de enriquecimento ou a marcação de espécies matrizes para coleta de 
sementes. As ações de manejo podem atuar em consonância com a capacidade de suporte do ambiente, tornando-se mais efetivas e condizentes com os mecanismos reguladores da formação florestal.

\section{CONCLUSÕES}

- O trecho de Mata Atlântica examinado apresenta padrão florístico típico de bordas de fragmentos florestais, com a participação de muitos indivíduos de poucas espécies, representados principalmente por plantas pioneiras, além de apresentar índice de Shannon $\left(\mathrm{H}^{\prime}\right)$ de 3,12 nats . ind. ${ }^{-1}$.

- Na comunidade em questão predominaram os padrões de dispersão agregado e uniforme. O primeiro é típico de formações florestais tropicais e o outro pode estar ligado a algum tipo de associação das espécies com determinado recurso do ambiente, tornando sua dispersão mais ampla.

- A aplicação do índice de Morisita pode ser de grande utilidade na conservação e no manejo florestal, pois a sua interpretação produz subsídios importantes para a elaboração de modelos de restauração de áreas degradadas, enriquecimentos de remanescentes florestais, conservação de germoplasma e outras atividades.

\section{AGRADECIMENTOS}

Os autores agradecem ao Sr. Roberto Lamêgo pela autorização de coleta de dados e hospedagem no Santuário de Vida Silvestre da Serra da Concórdia (SVSSC) (Valença - RJ).

\section{REFERÊNCIAS}

ANGIOSPERM PHYLOGENY GROUP. An update of the Angiosperm Phylogeny Group classification for the orders and families of flowering plants: APG III. Botanical Journal of the Linnean Society, v. 16, p. $105-121,2009$.

ARAÚJO, G. M.; GUIMARÃES, A. J. M.; NAKAJIMA, J. N. Fitossociologia de um remanescente de mata mesófila semidecídua urbana, Bosque John Kennedy, Araguari, MG, Brasil. Revista Brasileira de Botânica, v. 20, n. 1, p. 67 - 77, 1997.

ARRUDA, L.; DANIEL, O. Florística e diversidade em um fragmento de Floresta Estacional Semidecidual Aluvial em Dourados, MS. Revista Floresta, v. 37, n. 2, p.189 - 1999, 2007.

BERNASOL, W. P.; LIMA-RIBEIRO, M. S. Estrutura espacial e diamétrica de espécies arbóreas e seus condicionantes em um fragmento de cerrado sentido restrito no sudoeste goiano. Hoehnea, v. 37, n. 2, p. $181-198,2010$.

BÓREM, R. A. T.; OLIVEIRA FILHO, A. T. Fitossociologia do estrato arbóreo em uma toposeqüência alternada de Mata Atlântica, no Município de Silva Jardim - RJ, Brasil. Revista Árvore, v. 26, n. 6, p. $727-742,2002$.

BROWER, J. E.; ZAR, J. H. Field \& laboratory methods for general ecology. 2 ed. Iowa: Wm. C. Brown Publishers, 1984. 226 p.

BUDKE, J. C.; ATHAYDE, E. A.; GIEHL, E. L. H.; ZÁCHIA, R. A.; EISINGER, S. M. Composição florística e estratégias de dispersão de espécies lenhosas em uma floresta ribeirinha, arroio Passo das Tropas, Santa Maria, RS, Brasil. Iheringia, v. 60, n. 1, p. 17 - 24, 2005.

CAIN, S. A. The species-area curve. The American Midland Naturalist, v. 19, p. 573 - 581, 1938.

CAIN, M. L.; BOWMAN, W. D.; HACKER, S. D. Ecologia. Porto Alegre: Artmed, 2011. 640 p.

CALDAS, A. J. F. S. Geoprocessamento e análise ambiental para determinação de corredores de hábitat na Serra da Concórdia, Vale do Paraíba - RJ. 110 f. Dissertação (Mestrado em Ciências Ambientais e Florestais) - Instituto de Florestas, Universidade Federal Rural do Rio de Janeiro, Seropédica, 2006. 
CRAWLEY, M. J. Plant Ecology. Oxford: Blackwell Scientific Publications, 1997. 496 p.

DAN, M. L.; BRAGA, J. M. A.; NASCIMENTO, M. T. Estrutura da comunidade arbórea de fragmentos de Floresta Estacional Semidecidual na bacia hidrográfica do rio São Domingos, Rio de Janeiro, Brasil. Rodriguésia, v. 61, n. 4, p. 749 - 766, 2010.

DIETVORST, P.; MAAREL, E. VAN DER; PUTTEN, H. VAN DER. A new approach to the minimal area of a plant community. Vegetatio, v. 50, n. 2, p. 77 - 91, 1982.

DURIGAN, G.; FRANCO, G. A. D. C.; SAITO, M.; BAITELlO, J. B. Estrutura e diversidade do componente arbóreo da floresta na Estação Ecológica dos Caetetus, Gália, SP. Revista Brasileira de Botânica, v. 23, n. 4, p. 371 - 383, 2000.

FERNANDES, M. M.; CALDAS, A. J. F. S.; JIMENEZ, L. O. M.; CREPALDI, M. O. S.; BARBOZA, R. S.; RODRIGUES, R. M. M. Composição florística e estrutura do componente arbóreo de uma Floresta Estacional Semidecidual da fazenda Santa Cecília do Ingá, Volta Redonda, Rio de Janeiro, Brasil. Revista Científica Eletrônica de Engenharia Florestal, v. 20, n. 1, 2012. Disponível em: < http://faef.revista.inf.br/imagens_arquivos/arquivos_destaque/8H8Buz9tky2yVwk_2013-4-29-15-4226.pdf $>$. Acesso em: 14/01/2013.

FERREIRA, L. V.; LAURENCE, W. F. Effects of forest fragmentation on mortality and damage of selected trees in central Amazonia. Conservation Biology, v. 11, n. 3, p. 797 - 801, 1997.

FONSECA, R. C. B.; RODRIGUES, R. R. Análise estrutural e aspectos do mosaico sucessional de uma Floresta Semidecídua em Botucatu, SP. Scientia Forestalis, n. 57, p. 27 - 43, 2000.

FREITAS W. K.; MAGALHÃES L. M. S. Métodos e parâmetros para estudo da vegetação com ênfase no estrato arbóreo. Floresta e Ambiente, v. 19, n. 4, p. 520 - 540, 2012.

GENTRY, A. H. Changes in plant community diversity and floristic composition on environmental and geographical gradients. Ann. Missouri Bot. Gard., v. 75, p. 1 - 34, 1988.

GROMBONE, M. T.; BERNACCI, L. C.; NETO, J. A. A. M.; TAMASHIRO, J. Y.; LEITÃO FILHO, H. F. Estrutura fitossociológica da Floresta Semidecídua de Altitude do Parque municipal da Grota Funda Atibaia - SP. Acta Botanica Brasilica, v. 2, n. 4, p. 47 - 64, 1990.

GUEDES-BRUNI, R. R. Composição, estrutura e similaridade florística de dossel em seis unidades de Mata Atlântica no Rio de Janeiro. 231 f. Tese (Doutorado) - Instituto de Biociências da USP Universidade de São Paulo, São Paulo, 1998.

HAY, JOHN D.; BIZERRIL, M. X.; CALOURO, A. M.; COSTA, E. M. N.; FERREIRA, A. A.; GASTAL, M. L. A.; GOES JUNIOR, C. D.; MANZAN, D. J.; MARTINS, C. R.; MONTEIRO, J. M. G.; OLIVEIRA, S. A.; RODRIGUES, M. C. M.; SEYFFARTH, J. A. S.; WALTER, B. M. T. Comparação do padrão da distribuição espacial em escalas diferentes de espécies nativas do cerrado, em Brasília, DF. Revista Brasileira de Botânica, v. 23, n. 3, p. 341 - 347, 2000.

HUTCHINSON, G. E. The concept of pattern in ecology. Proceedings of the Academy of Natural Sciences of Philadelphia. 105, 1953. $12 \mathrm{p}$.

INSTITUTO BRASILEIRO DE GEOGRAFIA E ESTATÍSTICA (IBGE). Manual técnico da vegetação brasileira. Rio de Janeiro. Fundação Instituto Brasileiro de Geografia e Estatística, 1992. 92 p. (Série Manuais Técnicos em Geociências n 1).

INTERNATIONAL PLANT NAMES INDEX - IPNI. 2012. The International Plant Names Index. Disponível em < http://www.ipni.org/ > . Acesso em: 11/11/2012.

IVANAUSKAS, N. M.; RODRIGUES, R. R.; NAVE, A. G. Fitossociologia de um trecho de Floresta Estacional Semidecidual em Itatinga, São Paulo, Brasil. Scientia Forestalis, v. 56, p. 83 - 99, 1999.

KÖPPEN, W. Climatologia: con un estudio de los climas de la tierra. México: Fondo de Cultura Econômica, 1948. 479 p. 
LAMPRECHT, H. Silvicultura nos trópicos: ecossistemas florestais e respectivas espécies arbóreaspossibilidades e métodos de aproveitamento sustentado. Rossdorf: República Federal da Alemanha: Dt. Ges. Für Techn. Zusammenarbeit; 1990. 343 p.

LEGENDRE, P.; FORTIN, M. J. Spatial pattern and ecological analysis. Vegetatio, v. 80, n. 2, p. 107 $138,1989$.

LOPES, S. de F.; SCHIAVINI, I.; OLIVEIRA, A. P.; VALE, V. S. An Ecological Comparison of Floristic Composition in Seasonal Semideciduous Forest in Southeast Brazil: Implications for Conservation. International Journal of Forestry Research., v. 2012, 14 p. Disponível em: $<$ http://www.hindawi.com/journals/ijfr/2012/537269/ref/>. Acesso em: 10/12/2012.

MATTEUCCI, S. D.; COLMA, A. Metodologia para el estudio de la vegetacion. Washington: The Genral Secretarial of the Organization of American States, 1982. 167 p. (Série Biologia - Monografia, 22).

MISSOURI BOTANICAL GARDEN - MOBOT. 2012. Explore the beta release of Web TROPICOS. Disponível em < http://www.tropicos.org/ > . Acesso em: 11/11/2012.

MOREIRA, F. M. S. Fixação Biológica do Nitrogênio em espécies arbóreas. In: ARAUJO, R. S. \& HUNGRIA, M. (eds.). Microrganismos de importância Agrícola. Brasília: EMBRAPA-SPI, 1994. p. $121-149$.

MORISITA, M. Id--index, a measure of dispersion of individuals. Researches on Population Ecology, v. 4, p. 1 - 7, 1962.

OLIVEIRA, A. N.; AMARAL, I. L. Aspectos florísticos, fitossociológicos e ecológicos de um subbosque de terra firme na Amazônia Central, Amazonas, Brasil. Acta Amazônica, v. 35, n. 1, p. 1 - 16, 2005.

OLIVEIRA-FILHO, A. T.; FONTES, M. A. L. Patterns of floristic differentiation among Atlantic Forest in Southeastern Brazil and the influence of climate. Biotropica, v. 32, p. 793 - 810, 2000.

OLIVEIRA-FILHO, A. T.; JARENKOW, J. A.; RODAL, M. J. N. Floristic relationships of seasonally dry forests of Eastern South America based on tree species distribution patterns. In: R.T PENNINGTON; G. P. LEWIS; J. A. RATTER (eds.). Neotropical savannas and dry forests: plant diversity, biogeography, and conservation. Oxford: Taylor \& Francis CRC Press, 2006, p. 159 - 192.

PEIXOTO, A. L.; GENTRY, A. Diversidade e composição florística da mata de tabuleiro na Reserva Florestal de Linhares (Espírito Santo, Brasil). Revista Brasileira de Botânica, v. 13, n. 1, p. 19 - 25, 1990.

PIELOU, E. C. 'Shannon's formula as a measure of species diversity: its use and misuse'. The American Naturalist, v. 100, p. 463 - 465, 1966.

PINTO SOBRINHO, F. A.; CHRISTO, F. G.; GUEDES-BRUNI, R. R. fitossociologia do componente arbóreo num remanescente de Floresta Ombrófila Densa Submontana limítrofe à Reserva Biológica do Tinguá, Rio de Janeiro. Revista Floresta, v. 40, n. 1, p. 111 - 124, 2010.

POLHILL, R. M.; RAVEN, P. H.; STIRTON, C. H. Evolution and systematics of the Leguminosae. In: R. M. POLHILL; P.H. RAVEN (eds.). Advances in Legume Systematics. Kew, Royal Botanic Gardens. 1981. p. 1 - 26.

POOLE, R.W. An Introduction to Quantitative Ecology. New York: McGraw-Hill, 1974. 532 p.

PRADO, D. E.; GIBBS, P. E. Patterns of species distribution in the dry seasonal forests of South America. Annals of the Missouri Botanical Garden, 80, 902, 1993.

RODRIGUES, R. M. M.; MAGAlHÃES, L. M. S. Estrutura e Florística de Fragmento de Floresta Secundária na Planície Aluvionar do Rio Guandu, em Seropédica-RJ. Floresta \& Ambiente, v. 18, n. 3, p. $324-333,2011$. 
RODRIGUES, L. A.; CARVAlHO, D. A.; OliVEIRA FilHO, A. T.; BOTREL, R. T.; SilVA, E. A. Florística e estrutura da comunidade arbórea de um fragmento florestal em Luminárias, MG. Acta Botanica Brasílica, v. 17, n. 1, 71 - 97. 2003.

ROLIM, S. G.; NASCIMENTO, H. E. M. Análise da riqueza, diversidade e relação espécie-abundância de uma comunidade arbórea tropical em diferentes intensidades amostrais. Scientia Forestalis, v. 52, p. 7 - 16, 1997.

SCUDELLER, V. V.; MARTINS, F. R.; SHEPHERD, G. J. Distribution and abundance of arboreal species in the atlantic ombrophilous dense forest in Southeastern Brazil. Plant Ecology, v. 152, n. 2, p. 185 - 199, 2001.

SILVA, A. F.; LEITÃO FILHO, H. de F. Composição florística e estrutura de um trecho da Mata Atlântica de encosta no município de Ubatuba (São Paulo, Brasil). Revista Brasileira de Botânica, v. 5, n. $1 / 2$, p. $43-52,1982$.

SILVA, G. C.; NASCIMENTO, M. T. Fitossociologia de um remanescente de mata sobre tabuleiros no norte do estado do Rio de Janeiro (Mata do Carvão). Revista Brasileira de Botânica, v. 24, n. 1, p. 51 $62,2001$.

SILVA, K. E.; MARTINS, S. V.; SANTOS, N. T.; RIBEIRO, C. A. A. S. Padrão espacial de espécies arbóreas tropicais. In: MARTINS, S. V (Ed). Ecologia de Florestas Tropicais. Viçosa: Ed. UFV, 2009. p. $216-241$.

SILVA, M. A.; MELlO, J. M.; SCOLFORO, J. R. S.; CZANCK JÚNIOR, L.; ANDRADE, I. S.; OLIVEIRA, A. D. Análise da distribuição espacial da candeia (Eremanthus erythropappus (DC.) MacLeish) sujeita ao sistema de manejo porta-sementes. Cerne, v. 14, n. 4, p. 311 - 316, 2008.

SOUZA, G. R.; PEIXOTO, A. L.; FARIA, M. J. B.; ZAÚ, A. S. Composição florística e aspectos estruturais do estrato arbustivo-arbóreo de um trecho de Floresta Atlântica no médio vale do rio Paraíba do Sul, Rio de Janeiro, Brasil. Sitientibus, v. 7, n. 4, p. 398 - 409, 2007.

SPOLIDORO, M. L. C. V. Composição e estrutura de um trecho de floresta no médio Paraíba do Sul, RJ. 90 f. Dissertação (Mestrado em Ciências Ambientais e Florestais) - Instituto de Florestas, Universidade Federal Rural do Rio de Janeiro, Seropédica, 2001.

ZAÚ, A. S. Fragmentação da Mata Atlântica: aspectos teóricos. Floresta \& Ambiente, v. 6, n.1, p. 160 170, 1998. 
